\title{
Recurrence Relations for Hypergeometric Functions of Unit Argument
}

\section{By Stanisław Lewanowicz}

Abstract. We show that the generalized hypergeometric function

$$
P_{n}:={ }_{p+3} F_{p+2}\left(\begin{array}{c}
-n, n+\lambda, a_{p}, 1 \\
b_{p+2}
\end{array} \mid 1\right) \quad(n \geqslant 0)
$$

satisfies a nonhomogeneous recurrence relation of order $p+\sigma$, where $\sigma=0$ when ${ }_{p+3} F_{p+2}(1)$ is balanced, and $\sigma=1$ otherwise. Also, for

$$
U_{n}:=\frac{\left(c_{q+1}\right)_{n}}{\left(d_{q}\right)_{n}(n+\lambda)_{n}{ }_{q+2}} F_{q+1}\left(\begin{array}{c}
n+c_{q+2} \\
n+d_{q}, 2 n+\lambda+1
\end{array}\right) \quad(n \geqslant 0)
$$

a homogeneous recurrence relation of order $q+1$ is given.

1. Notation. The generalized hypergeometric series

$$
{ }_{p+1} F_{p}\left(\begin{array}{c}
a_{1}, a_{2}, \ldots, a_{p+1} \\
b_{1}, b_{2}, \ldots, b_{p}
\end{array} \mid z\right)=\sum_{k=0}^{\infty}\left[\prod_{i=1}^{p+1}\left(a_{i}\right)_{k} z^{k} / k ! \prod_{j=1}^{p}\left(b_{j}\right)_{k}\right],
$$

where

$$
(\alpha)_{k}:=\Gamma(\alpha+k) / \Gamma(\alpha),
$$

converges for $|z|<1$. Further, this series is absolutely convergent for $|z|=1$ if

$$
\operatorname{Re}\left(\sum_{i=1}^{p+1} a_{i}-\sum_{j=1}^{p} b_{j}\right)<0 .
$$

However, if any $a_{i}$ is zero or a negative integer, then (1.1) always converges, since it terminates. If any $b_{j}$ is 0 or a negative integer, the series is not defined. If $a_{i}=b_{j}$ for some $i$ and $j, 1 \leqslant i \leqslant p+1,1 \leqslant j \leqslant p$, then ${ }_{p+1} F_{p}$ reduces to ${ }_{p} F_{p-1}$.

If in (1.1) $z=1$, and the parameters are such that

$$
1+\sum_{i=1}^{p+1} a_{i}=\sum_{j=1}^{p} b_{j}
$$

then the ${ }_{p+1} F_{p}$ is said to be balanced.

Throughout, we employ a contracted notation. Given the function $M$, we define

$$
M\left(a_{p+1}\right):=\prod_{i=1}^{p+1} M\left(a_{i}\right), \quad M\left(b_{p}\right):=\prod_{j=1}^{p} M\left(b_{j}\right) .
$$

Received November 16, 1983; revised April 17, 1984.

1980 Mathematics Subject Classification. Primary 33A30, 65D20, 65Q05.

(c)1985 American Mathematical Society $0025-5718 / 85 \$ 1.00+\$ .25$ per page 
The symbol $1+a_{h}-a_{p+1}^{*}$ stands for the set $1+a_{h}-a_{1}, 1+a_{h}-a_{2}, \ldots, 1+a_{h}$ $-a_{h-1}, 1+a_{h}-a_{h+1}, \ldots, 1+a_{h}-a_{p+1}$. Thus we may write (1.1) in abbreviated form

$$
{ }_{p+1} F_{p}\left(\begin{array}{c}
a_{p+1} \\
b_{p}
\end{array} \mid z\right)=\sum_{k=0}^{\infty}\left[\left(a_{p+1}\right)_{k} z^{k} / k !\left(b_{p}\right)_{k}\right] .
$$

For a treatment of the generalized hypergeometric functions, see any of the references [1], [4], [5].

2. Introduction. In this paper we derive new difference equations satisfied by the functions

$$
\begin{gathered}
P_{n}:={ }_{p+3} F_{p+2}\left(\begin{array}{c}
-n, n+\lambda, a_{p}, 1 \\
b_{p+2}
\end{array} \mid 1\right) \\
U_{n}:=\frac{\left(c_{q+1}\right)_{n}}{\left(d_{q}\right)_{n}(n+\lambda)_{n}{ }_{q+2}} F_{q+1}\left(\begin{array}{c}
n+c_{q+2} \\
n+d_{q}, 2 n+\lambda+1
\end{array}\right) \\
\quad(n=0,1, \ldots) .
\end{gathered}
$$

Notice that recursion relationships of the forms

$$
\begin{aligned}
& \sum_{m=0}^{p+2} G_{m}(n ; p+2) \Phi_{n-m}=\gamma(n ; p+2), \\
& \sum_{m=0}^{q+2}(-1)^{m} H_{m}(n ; q+2) \Psi_{n+m}=0,
\end{aligned}
$$

where $G_{m}(n ; t), H_{m}(n ; t), \gamma(n ; t)$ are rational in $n$ (see formulae (3.1), (3.2) and (3.11) below), are satisfied by (2.1) and (2.2), respectively. The relations are of order $p+2$ and $q+2$, respectively. This result can be deduced from some general theorems due to Wimp [8] (see also [4, vol. 2, p. $135 \mathrm{ff}$.$] ).$

We show that (2.1) also obeys the difference equation

$$
\sum_{m=0}^{p+\sigma} G_{m}(n ; p+\sigma) \Phi_{n-m}=\gamma(n ; p+\sigma),
$$

of order $p+\sigma$, where $\sigma=0$ for the balanced case,

$$
\lambda+2+\sum_{i=1}^{p} a_{i}=\sum_{j=1}^{p+2} b_{j},
$$

and $\sigma=1$ for the general case. Further, we show that (2.2) obeys the equation

$$
\sum_{m=0}^{q+1}(-1)^{m} H_{m}(n ; q+1) \Psi_{n+m}=0
$$

of order $q+1$. Notice that the coefficients of Eqs. (2.3) and (2.5) are formed according to the same general rule (3.1). A similar statement is true for the coefficients of (2.4), (2.6) and for formula (3.2).

For applications, it is convenient to have available the functions which satisfy the same difference equation as does $P_{n}$ ( $U_{n}$, respectively), or the homogeneous form of this equation. A theorem, given in the next section, also provides information of this kind. 
3. Results. Let $p, q, n, m, t$ be integers $\geqslant 0$. Let $\lambda, a_{i}(i=1,2, \ldots, p), b_{j}(j=$ $1,2, \ldots, p+2), c_{k}(k=1,2, \ldots, q+2), d_{l}(l=1,2, \ldots, q)$ be complex constants. We define the functions

$$
\begin{aligned}
& G_{m}(n ; t):=\frac{(n+1-m)_{m}(2 n+\lambda-2 m)_{2 m}}{(n+\lambda-m)_{m}(2 n+\lambda-m-t)_{m}\left(n+b_{p+2}-1\right)} \\
& \times\left\{\frac{1}{m !}\left(n-m-1+b_{p+2}\right)\right. \\
& \times_{p+4} F_{p+3}\left(\begin{array}{c}
-m, 2 n+\lambda-m-t, n-m+b_{p+2} \\
2 n+\lambda-2 m+1, n-m-1+b_{p+2}
\end{array} \mid 1\right) \\
& +\frac{1}{\Gamma(m)}(2 n+\lambda-m-t)\left(n-m+a_{p}\right) \\
& \left.\times_{p+2} F_{p+1}\left(\begin{array}{c}
1-m, 2 n+\lambda-t+1, n-m+1+a_{p} \\
2 n+\lambda-2 m+1, n-m+a_{p}
\end{array} \mid 1\right)\right\}, \\
& H_{m}(n ; t):=\frac{(2 n+\lambda)_{m}(n+\beta+1)_{m}}{(n+\lambda)_{m}} \\
& \times\left\{\frac{1}{m !}{ }_{q+4} F_{q+3}\left(\begin{array}{c}
-m, n+2 n+\lambda, n+1+c_{q+2} \\
2 n+\lambda+q+2, n+c_{q+2}
\end{array} \mid 1\right)\right. \\
& +\frac{(2 n+\lambda+m)\left(n+d_{q}\right)}{\Gamma(m)\left(n+c_{q+2}\right)} \\
& \left.\times_{q+2} F_{q+1}\left(\begin{array}{c}
1-m, 2 n+\lambda+m+1, n+1+d_{q} \\
2 n+\lambda+q+2, n+d_{q}
\end{array} \mid 1\right)\right\} .
\end{aligned}
$$

Here $\beta+1=c_{i}$ for $i=q+2$. We assume the parameters to be such that all expressions make sense. By applying a lemma of Wimp [8], we get the alternative forms for $G_{m}, H_{m}$ :

$$
\begin{aligned}
G_{m}(n ; t) & =\frac{(-1)^{p}(n+1-m)_{m}(2 n+\lambda-2 m)(2 n+\lambda-t+1)_{t-1}}{(n+\lambda-m)_{m}(2 n+\lambda-m-t)_{m}\left(n-1-b_{p+2}\right)} \\
& \times\left\{\frac{1}{(t-m) !}\left(n+\lambda-t+1-b_{p+2}\right)\right.
\end{aligned}
$$

$$
\begin{aligned}
& \times_{p+4} F_{p+3}\left(\begin{array}{c}
m-t, 2 n+\lambda-m-t, n+\lambda-t+2-b_{p+2} \\
2 n+\lambda-t+1, n+\lambda-t+1+b_{p+2}
\end{array} \mid 1\right) \\
& +\frac{1}{\Gamma(t-m)}(2 n+\lambda-m-t)\left(n+\lambda-t+1-a_{p}\right) \\
& \left.\times_{p+2} F_{p+1}\left(\begin{array}{c}
m-t+1,2 n+\lambda-m-t+1, n+\lambda-t+2-a_{p} \\
2 n+\lambda-t+1, n+\lambda-t+1-a_{p}
\end{array} \mid 1\right)\right\},
\end{aligned}
$$




$$
\begin{aligned}
H_{m}(n ; t)= & \frac{(-1)^{q}(2 n+\lambda)_{q+2}(n+\beta+1)_{m}}{(n+\lambda)_{m}(2 n+\lambda+m)_{m+1}\left(n+c_{q+2}\right)} \\
& \times\left\{\frac{\left(n+\lambda+m-c_{q+2}\right)}{(t-m) !}\right. \\
& \quad{ }_{q+4} F_{q+3}\left(\begin{array}{c}
m-t, 2 n+\lambda+m, n+m+\lambda+1-c_{q+2} \\
2 n+\lambda+2 m+1, n+\lambda+m-c_{q+2}
\end{array} \mid 1\right) \\
& +\frac{1}{\Gamma(t-m)}(2 n+\lambda+m)\left(n+\lambda+m+1-d_{q}\right) \\
& \left.\quad \times{ }_{q+2} F_{q+1}\left(\begin{array}{c}
m-t+1,2 n+\lambda+m+1, n+m+\lambda+2-d_{q} \\
2 n+\lambda+2 m+1, n+m+\lambda+1-d_{q}
\end{array}\right)\right\} .
\end{aligned}
$$

In particular, we have

$$
\begin{aligned}
& G_{0}(n ; t)=H_{0}(n ; t) \equiv 1 \\
& G_{t}(n ; t)=\frac{(-1)^{p}(n+1-t)_{t}(2 n+\lambda-t+1)_{t-1}\left(n+\lambda-t+1-b_{p+2}\right)}{(n+\lambda-t)_{t}(2 n+\lambda-2 t+1)_{t-1}\left(n-1+b_{p+2}\right)} \\
& H_{t}(n ; t)=\frac{(-1)^{q}(2 n+\lambda)_{q+2}(n+\beta+1)_{t}\left(n+\lambda+t-c_{q+2}\right)}{(n+\lambda)_{t}(2 n+\lambda+t)_{t+1}\left(n+c_{q+2}\right)}
\end{aligned}
$$

Observe that

$$
{ }_{r+2} F_{r+1}\left(\begin{array}{c}
-m, m+\alpha, 1+\omega_{r} \mid 1 \\
k+\alpha, \omega_{r}
\end{array}\right)=0 \quad(m, k=0,1, \ldots)
$$

for $m \geqslant k>r$ (see Luke [4, vol. 1, p. 114]). This shows that $G_{m}(n ; t)=0$ for $m \geqslant t+1>p+2$, and $H_{m}(n ; t)=0$ for $m \geqslant t+1>q+2$.

Let

$$
G_{m}^{*}(n ; t):=(n+\lambda-t)_{t}(2 n+\lambda-2 t+1)_{t-1}\left(n+b_{p+2}-1\right) G_{m}(n ; t) .
$$

Using (3.1), (3.3) and an identity from Luke [4, vol. 1, p. 42], one checks that

$$
G_{t-m}^{*}(t-n-\lambda ; t)=-G_{m}^{*}(n ; t) \quad(m=0,1, \ldots, t) .
$$

Similarly, for

$$
\begin{aligned}
H_{m}^{*}(n ; t):= & (n+\lambda)_{m}(n+m+\lambda-\beta)_{t-m} \\
& \times(2 n+\lambda+t+1)_{t}\left(n+c_{q+2}\right) H_{m}(n ; t),
\end{aligned}
$$

we have

$$
H_{t-m}^{*}(-n-\lambda-t ; t)=H_{m}^{*}(n ; t) \quad(m=0,1, \ldots, t) .
$$

We need the following result, which can be easily obtained with the aid of some formulae from Luke [4, vol. 1, p. 26]. If $m \geqslant r \geqslant 0, m$ and $r$ integers, then

$$
{ }_{r+2} F_{r+1}\left(\begin{array}{c}
-m, m+\alpha, 1+\omega_{r} \\
r-h+\alpha, \omega_{r}
\end{array} \mid 1\right)=\frac{(-1)^{m} m ! \Gamma(r-h+\alpha)}{\Gamma(m+\alpha) \omega_{r}} s_{h}
$$


for $h=0,1$, where

$$
s_{0}:=1, \quad s_{1}:=\frac{1}{2}(m)_{2}+\frac{1}{2}(m-r+1)(m+r+2 \alpha-2)+\sum_{i=1}^{r} \omega_{i} .
$$

For $n, t$ integers $\geqslant 0$, we define

$$
\gamma(n ; t):=\frac{\left(b_{p+2}-1\right)(n+\lambda)_{n}}{\left(n+b_{p+2}-1\right)(n+\lambda-t)_{n}} .
$$

THEOREM. The function

$$
P_{n}:={ }_{p+3} F_{p+2}\left(\begin{array}{c}
-n, n+\lambda, a_{p}, 1 \\
b_{p+2}
\end{array} \mid 1\right)
$$

satisfies the difference equation

$$
\sum_{m=0}^{p+\sigma} G_{m}(n ; p+\sigma) \Phi_{n-m}=\gamma(n ; p+\sigma) \quad(n \geqslant p+\sigma),
$$

where the functions $G_{m}$ and $\gamma$ are defined by (3.1) (or (3.3)) and (3.11), respectively, and

$$
\sigma:=\operatorname{sgn}\left(\left\|\lambda+2+\sum_{i=1}^{p} a_{i}-\sum_{j=1}^{p+2} b_{j}\right\|\right. \text {. }
$$

Moreover, if

$$
\operatorname{Re}\left(\lambda+\sum_{i=1}^{p} a_{i}-\sum_{j=1}^{p+2} b_{j}\right)<-2
$$

then another solution of (3.13) with $\sigma=1$ is

$$
\begin{aligned}
Q_{n} & :=\frac{b_{p+2}-1}{(n+1)(n+\lambda-1)\left(a_{p}-1\right)^{p+3}} F_{p+2}\left(\begin{array}{c}
2-b_{p+2}, 1 \\
n+2,2-n-\lambda, 2-a_{p}
\end{array} \mid 1\right) \\
& =1-{ }_{p+3} F_{p+2}\left(\begin{array}{c}
1-b_{p+2}, 1 \\
n+1,1-n-\lambda, 1-a_{p}
\end{array} \mid 1\right) .
\end{aligned}
$$

Finally, if

$$
\operatorname{Re}\left(\lambda+\sum_{i=1}^{p} a_{i}-\sum_{j=1}^{p+2} b_{j}\right)<-1
$$

then the functions

$$
R_{n}^{(j)}:=\frac{\Gamma(n+1) \Gamma\left(n+\lambda-a_{j}\right)}{\Gamma\left(n+1+a_{j}\right) \Gamma(n+\lambda)}
$$

$$
\begin{aligned}
\times_{p+2} F_{p+1}\left(\begin{array}{c}
1+a_{j}-b_{p+2} \\
1+a_{j}+n, 1+a_{j}-n-\lambda, 1+a_{j}-a_{p}^{*}
\end{array} \mid 1\right) & (j=1,2, \ldots, p), \\
R_{n}^{(p+1)}:= & \frac{\Gamma(n+1) \Gamma(1-n-\lambda) \Gamma(2 n+\lambda) \Gamma\left(n+a_{p}\right)}{\Gamma\left(n+b_{p+2}\right)} \\
& \times_{p+2} F_{p+1}\left(\begin{array}{c}
1-n-b_{p+2} \\
1-\lambda-2 n, 1-n-a_{p}
\end{array} \mid 1\right),
\end{aligned}
$$




$$
\begin{aligned}
R_{n}^{(p+2)}:= & \frac{\Gamma(1-n-\lambda) \Gamma(n+1) \Gamma\left(n+\lambda+1-b_{p+2}\right)}{\Gamma(2 n+\lambda+1) \Gamma\left(n+\lambda+1-a_{p}\right)} \\
& \times_{p+2} F_{p+1}\left(\begin{array}{c}
n+\lambda+1-b_{p+2} \\
2 n+\lambda+1,1+n+\lambda-a_{p}
\end{array} \mid 1\right)
\end{aligned}
$$

under the condition that no two of the quantities $-n, n+\lambda, a_{i}(i=1,2, \ldots, p)$ differ by an integer or zero, and the functions

$$
\begin{aligned}
S_{n}^{(k)}:= & \frac{\Gamma(n+1) \Gamma\left(n+\lambda+1-b_{k}\right)}{\Gamma\left(n+b_{k}\right) \Gamma(n+\lambda)} \\
& \times_{p+2} F_{p+1}\left(\begin{array}{c}
1-b_{k}-n, 1-b_{k}+n+\lambda, 1-b_{k}+a_{p} \mid \\
1-b_{k}+b_{p+2}^{*} \\
1
\end{array}\right)
\end{aligned}
$$

under the condition that no two of the parameters $b_{j}(j=1,2, \ldots, p+2)$ differ by an integer or zero, satisfy the difference equation

$$
\sum_{m=0}^{p+1} G_{m}(n ; p+1) \Phi_{n-m}=0 \quad(n \geqslant p+1) .
$$

Remarks. $1^{\circ}$. It follows from a theorem in [4, vol. 2, p. 136], that (3.12), (3.15) satisfy Eq. (2.3), while the functions (3.16)-(3.19) are solutions of the homogeneous form of this equation. $2^{\circ}$. That the function (3.12) with $b_{i}=1$ for $i=p+2$ satisfies a homogeneous equation of order $p+1$ (Eq. (3.20), in fact) follows from results of Wimp [10]. (Note that formulae (5), (6) of [10] should be corrected by multiplying the $s$ th term of each sum by $1 / s$ ! (see [12]).) $3^{\circ}$. It can be conjectured that if no $a_{i}$ is equal to any $b_{j}$, the functions (3.12), (3.15) do not satisfy a nontrivial equation of the form (3.13) of lower order than $p+\sigma$ or $p+1$, respectively, and none of the functions (3.16)-(3.19) satisfy an equation of the form (3.20) of lower order than $p+1$. (We assume that $a_{i}, b_{j}$ are not interrelated.)

Proof. A. Putting the function (3.12) in the left-hand side of (3.13), turning the sum around, replacing $n$ by $n+p+\sigma$, and performing some algebra, we get

$$
\sum_{m=0}^{p+\sigma} G_{p+\sigma-m}(n+p+\sigma ; p+\sigma) P_{n+m}=\gamma(n+p+\sigma ; p+\sigma)\left(\phi_{n}+\psi_{n}\right),
$$

where

$$
\begin{aligned}
\phi_{n}:= & \sum_{k=n}^{n+p+\sigma} \xi_{k}, \quad \psi_{n}:=\frac{(n+p+\sigma)(n+\lambda)\left(a_{p}\right)}{b_{p+2}-1} \sum_{k=n}^{n+p+\sigma-1} \eta_{k}, \\
\xi_{k}:= & \frac{(-1)^{k}(-n-p-\sigma)_{k}(n+\lambda)_{k}\left(b_{p+2}\right)_{k}}{k !\left(b_{p+2}-1\right)_{k}(k+\lambda)_{k}} \\
& \times_{p+4} F_{p+3}\left(\begin{array}{c}
k-n-p-\sigma, k+n+\lambda, k+b_{p+2} \mid \\
2 k+\lambda+1, k-1-b_{p+2}
\end{array} \mid 1\right) \\
& \times_{p+3} F_{p+2}\left(\begin{array}{c}
-k, k+\lambda, a_{p}, 1 \\
b_{p+2}
\end{array} \mid 1\right) \quad(k \geqslant 0),
\end{aligned}
$$




$$
\begin{aligned}
\eta_{k}:= & \frac{(-1)^{k}(1-n-p-\sigma)_{k}(n+\lambda+1)_{k}\left(1+a_{p}\right)_{k}}{k !(k+\lambda)_{k}\left(a_{p}\right)_{k}} \\
& \times_{p+2} F_{p+1}\left(\begin{array}{c}
k-n-p-\sigma+1, k+n+\lambda+1, k+1+a_{p} \mid \\
2 k+\lambda+1, k+a_{p}
\end{array}\right) \\
& \times_{p+3} F_{p+2}\left(\begin{array}{c}
-k, k+\lambda, a_{p}, 1 \\
b_{p+2}
\end{array} \mid 1\right) \quad(k \geqslant 0) .
\end{aligned}
$$

Let

$$
f_{n}:={ }_{p+3} F_{p+2}\left(\begin{array}{c}
-n-p-\sigma, n+\lambda, a_{p}, 1 \\
b_{p+2}-1
\end{array} \mid 1\right) .
$$

By applying a theorem in [4, vol. 2, p. 7], we have

$$
f_{n}=\sum_{k=0}^{n+p+\sigma} \xi_{k}=\phi_{n}+\rho_{n}
$$

where

Similı rly, for

$$
\rho_{n}:=\sum_{k=0}^{n-1} \xi_{k}
$$

$$
\begin{aligned}
f_{n}^{*}: & ={ }_{p+3} F_{p+2}\left(\begin{array}{c}
1-n-p-\sigma, n+\lambda+1,1+a_{p}, 1 \\
b_{p+2}
\end{array} \mid 1\right) \\
& =\frac{b_{p+2}-1}{(n+p+\sigma)(n+\lambda) a_{p}}\left(1-f_{n}\right),
\end{aligned}
$$

by virtue of the same theorem, we obtain

which implies

$$
f_{n}^{*}=\sum_{k=0}^{n+p+\sigma-1} \eta_{k}
$$

where

$$
1-f_{n}=\psi_{n}+\tau_{n}
$$

$$
\tau_{n}:=\frac{(n+p+\sigma)(n+\lambda) a_{p}}{b_{p+2}-1} \sum_{k=0}^{n-1} \eta_{k} .
$$

Applying (3.10), we get for $k=0,1, \ldots, n-1$, the equations

$$
\begin{aligned}
& { }_{p+4} F_{p+3}\left(\begin{array}{c}
-h, h+\mu, k+b_{p+2} \\
\mu+p+\sigma+1, k-1-b_{p+2}
\end{array} \mid 1\right)=\frac{(-1)^{h} h ! \Gamma(2 k+\lambda+1)}{\Gamma(k+n+\lambda)\left(k-1+b_{p+2}\right)} y_{k \sigma}, \\
& { }_{p+2} F_{p+1}\left(\begin{array}{c}
1-h, h-1+\nu, k+1+a_{p} \\
\nu+p+\sigma-1, k+a_{p}
\end{array} \mid 1\right)=\frac{(-1)^{h-1}(h-1) ! \Gamma(2 k+\lambda+1)}{\Gamma(k+n+\lambda+1)\left(k+a_{p}\right)} z_{k \sigma},
\end{aligned}
$$

where

$$
\begin{aligned}
& h:=n+p+\sigma-k, \quad \mu:=2 k+\lambda-p-\sigma, \quad \nu:=\mu+2, \\
& y_{k 1}:=z_{k 1}:=1, \quad y_{k 0}:=w_{k}+\sum_{i=1}^{p+2} b_{i}, \quad z_{k 0}:=w_{k}+\lambda+2+\sum_{i=1}^{p} a_{i}, \\
& w_{k}:=(k-1)(p+2)+\frac{1}{2}(n+p-k)_{2}+\frac{1}{2}(n-k-1)(n+3 k+2 \lambda) .
\end{aligned}
$$


Hence

$$
\rho_{n}=\delta \sum_{k=0}^{n-1} \omega_{k} y_{k \sigma}, \quad \tau_{n}=-\delta \sum_{k=0}^{n-1} \omega_{k} z_{k \sigma}
$$

where

$$
\begin{gathered}
\delta:=\frac{(-1)^{p+n+\sigma}(p+n+\sigma) !}{\Gamma(n+\lambda)\left(b_{p+2}-1\right)}, \\
\omega_{k}:=\frac{(-1)^{k}(2 k+\lambda) \Gamma(k+\lambda)}{k !}{ }_{p+3} F_{p+2}\left(\begin{array}{c}
-k, k+\lambda, a_{p}, 1 \\
b_{p+2}
\end{array} \mid 1\right) \\
\quad(k=0,1, \ldots, n-1) .
\end{gathered}
$$

As, in view of the definition (3.14), $y_{k \sigma}=z_{k \sigma}$ for $\sigma=0,1$ and $k=0,1, \ldots, n-1$, we obtain $\rho_{n}=-\tau_{n}$. This, together with (3.21)-(3.23), implies that the function (3.12) satisfies Eq. (3.13).

B. A similar argument shows that the function (3.15) satisfies Eq. (3.13) with $\sigma=1$. Putting $Q_{n}$ in the left-hand side of (3.13), we get after some algebra

$$
\sum_{m=0}^{p+1} G_{p+1-m}(n+p+1 ; p+1) Q_{n+m}=\gamma(n+p+1 ; p+1)\left(\phi_{n}+\psi_{n}\right),
$$

where

$$
\begin{aligned}
\phi_{n}:= & \left(n+\lambda+1-b_{p+2}\right) \kappa_{n} \\
& \times \sum_{m=0}^{p+1} \mu_{m}(n)_{p+4} F_{p+3}\left(\begin{array}{c}
-m, m+2 n+\lambda, n+\lambda+2-b_{p+2} \\
2 n+\lambda+p+2, n+\lambda+1-b_{p+2}
\end{array} \mid 1\right), \\
\psi_{n}:= & \left(n+\lambda+1-a_{p}\right) \kappa_{n}
\end{aligned}
$$

$$
\begin{gathered}
\times \sum_{m=0}^{p} \mu_{m}(n+1)_{p+2} F_{p+1}\left(\begin{array}{c}
-m, m+2 n+\lambda+2, n+\lambda+2-a_{p} \\
2 n+\lambda+p+2, n+\lambda+1-a_{p}
\end{array} \mid 1\right), \\
\mu_{m}(n):=\frac{\Gamma(m+n+\lambda-1) \Gamma(m+2 n+\lambda)(2 m+2 n+\lambda)}{m ! \Gamma(m+n+2)} \\
\quad \times_{p+3} F_{p+2}\left(\begin{array}{c}
m+n+2,2-n-\lambda-m, 2-a_{p}
\end{array} \mid 1\right) \\
\kappa_{n}:=\frac{\Gamma(n+p+2)}{\left(1-a_{p}\right) \Gamma(n+\lambda) \Gamma(2 n+\lambda+p+2)} .
\end{gathered}
$$

Notice the use of the second form, i.e. (3.3), for $G_{m}$. By applying a theorem from [4, vol. 2, p. 5], and making use of (3.10), we obtain the equations

$$
\phi_{n}=\frac{2-b_{p+2}}{\left(1-a_{p}\right)(n+\lambda-1)(n+p+2)} g_{n}^{*}-\rho_{n}=1-g_{n}-\rho_{n}, \quad \psi_{n}=g_{n}+\rho_{n} \text {, }
$$


where

$$
\begin{gathered}
g_{n}:={ }_{p+3} F_{p+2}\left(\begin{array}{c}
2-b_{p+2}, 1 \\
n+p+2,1-n-\lambda, 1-a_{p} \mid 1
\end{array}\right), \\
\quad 3-b_{p+2}, 1 \\
g_{n}^{*}:={ }_{p+3} F_{p+2}\left(\begin{array}{c}
\mid \\
n+p+3,2-n-\lambda, 2-a_{p}
\end{array}\right), \\
\rho_{n}:=\Gamma(2 n+\lambda+p+2) \kappa_{n} \sum_{m=p+2}^{\infty}(-1)^{m} \frac{m ! \mu_{m}(n)}{\Gamma(m+2 n+\lambda)} .
\end{gathered}
$$

Hence (3.24) is equivalent to (3.13) with $\Phi_{n}=Q_{n}$ and $\sigma=1$.

C. We check that the functions (3.16) obey Eq. (3.20). Let $j$ be any integer from the set $\{1,2, \ldots, p\}$. Proceeding as in part B of the proof, we get

$$
\sum_{m=0}^{p+1} G_{p+1-m}(n+p+1 ; p+1) R_{n+m}^{(j)}=\gamma(n+p+1 ; p+1)\left(\phi_{n}+\psi_{n}\right),
$$

where $\phi_{n}$ and $\psi_{n}$ are as given in (3.25), (3.26), in which, however, the functions $\kappa_{n}$ and $\mu_{m}(n)$ are defined anew by

$$
\begin{gathered}
\kappa_{n}:=\frac{\Gamma(n+p+2)}{\left(1-b_{p+2}\right) \Gamma(n+\lambda) \Gamma(2 n+\lambda+p+2)}, \\
\mu_{m}(n):=\frac{\Gamma\left(m+n+\lambda-a_{j}\right) \Gamma(m+2 n+\lambda)(2 m+2 n+\lambda)}{m ! \Gamma\left(m+n+1-a_{j}\right)} \\
\times_{p+2} F_{p+1}\left(\begin{array}{c}
1+a_{j}-b_{p+2} \\
1+a_{j}+n+m, 1+a_{j}-n-\lambda-m, 1+a_{j}-a_{p}^{*}
\end{array} \mid 1\right) .
\end{gathered}
$$

Applying the above-mentioned theorem from [4, vol. 2, p. 5], and then using identity (3.10), we may write

$$
\phi_{n}=\kappa_{n}\left(f_{n}-\rho_{n}\right), \quad \psi_{n}=\kappa_{n}\left(\omega_{n}-f_{n}+\rho_{n}\right),
$$

where

$$
\begin{aligned}
& f_{n}:=\frac{\left(1+a_{j}-b_{p+2}\right) \Gamma\left(n+\lambda-a_{j}\right) \Gamma(2 n+\lambda+p+2)}{\Gamma\left(n+p+2+a_{j}\right)} \\
& \times_{p+2} F_{p+1}\left(\begin{array}{c}
2+a_{j}-b_{p+2} \\
1+a_{j}-n-\lambda, 2+a_{j}+n+p, 1+a_{j}-a_{p}^{*}
\end{array} \mid 1\right), \\
& \rho_{n}:=\Gamma(2 n+\lambda+p+2) \sum_{m=p+2}^{\infty}(-1)^{m} \frac{m ! \mu_{m}(n)}{\Gamma(m+2 n+\lambda)}, \\
& \omega_{n}:=\left(n+\lambda+1-a_{p}\right) \\
& \times \sum_{m=0}^{\infty} \frac{(2 m+2 n+\lambda+2) \Gamma(m+2 n+\lambda+2) \Gamma\left(m+n+\lambda+1-a_{j}\right)}{m ! \Gamma\left(m+n+2+a_{j}\right)} \\
& \times_{p+2} F_{p+1}\left(\begin{array}{c}
-m, m+2 n+\lambda+2, n+\lambda+2-a_{p} \\
2 n+\lambda+p+2, n+\lambda+1-a_{p}
\end{array} \mid 1\right) \text {. }
\end{aligned}
$$


Now, applying the identity

$$
\sum_{m=0}^{\infty} \frac{(2 m+\gamma)(-\mu)_{m}}{m !(m+\gamma)_{\mu+1}}{ }_{p+2} F_{p+1}\left(\begin{array}{c}
-m, m+\gamma, c_{p}-\mu \\
\alpha, d_{p}-\mu
\end{array} \mid 1\right)=\frac{\left(d_{p}\right)_{-\mu}}{(\alpha)_{\mu}\left(c_{p}\right)_{-\mu}},
$$

which can be deduced from another theorem in [4, vol. 2, p. 1], we conclude, after making an identification of the parameters $(\gamma=2 n+\lambda+2, \alpha=2 n+\lambda+p+2$, $\mu=a_{j}-n-\lambda-1, c_{i}=1+a_{j}-a_{i}, d_{i}=a_{j}-a_{i}$ for $\left.i=1,2, \ldots, p\right)$, that $\omega_{n}=0$. The result then follows from (3.27) and (3.29).

D. We show that the function $R_{n}^{(p+2)}$, defined in (3.18), obeys (3.20). Putting (3.18) in the left-hand side of (3.20) yields

$$
\begin{aligned}
& \sum_{m=0}^{p+1} G_{p+1-m}(n+p+1 ; p+1) R_{n+m}^{(p+2)} \\
& \quad=\gamma(n+p+1 ; p+1)(-1)^{n} c \kappa_{n}\left(\phi_{n}^{*}+\psi_{n}^{*}\right),
\end{aligned}
$$

where $c$ is a constant, $\kappa_{n}$ is given by (3.28), and

$$
\begin{aligned}
\phi_{n}^{*}:= & \left(n+\lambda+1-b_{p+2}\right) \\
& \times \sum_{m=0}^{p+1} \nu_{m}(n)_{p+4} F_{p+3}\left(\begin{array}{c}
-m, m+2 n+\lambda, n+\lambda+2-b_{p+2} \\
2 n+\lambda+p+2, n+\lambda+1-b_{p+2}
\end{array} \mid 1\right) \\
\psi_{n}^{*}:= & -\left(n+\lambda+1-a_{p}\right) \\
& \times \sum_{m=0}^{p} \nu_{m}(n+1)_{p+2} F_{p+1}\left(\begin{array}{c}
-m, m+2 n+\lambda+2, n+\lambda+2-a_{p} \\
2 n+\lambda+p+2, n+\lambda+1-a_{p}
\end{array} \mid 1\right), \\
\nu_{m}(n):= & \frac{(-1)^{m}(2 m+2 n+\lambda) \Gamma(m+2 n+\lambda) \Gamma\left(m+n+\lambda+1-b_{p+2}\right)}{m ! \Gamma(2 m+2 n+\lambda+1) \Gamma\left(m+n+\lambda+1-a_{p}\right)} \\
& \times{ }_{p+2} F_{p+1}\left(\begin{array}{c}
2 m+n+\lambda+1-b_{p+2} \\
2 m+\lambda+1, m+n+\lambda+1-a_{p}
\end{array}\right) .
\end{aligned}
$$

By virtue of the theorem mentioned in part $A$ of the proof (see $[4$, vol. 2, p. 7]), and using identity (3.10), we get

$$
\phi_{n}^{*}=-\psi_{n}^{*}=f_{n}-\rho_{n},
$$

where

$$
\begin{aligned}
& f_{n}:=\frac{\Gamma\left(n+\lambda+2-b_{p+2}\right)}{\Gamma\left(n+\lambda+1-a_{p}\right)}{ }_{p+2} F_{p+1}\left(\begin{array}{c}
n+\lambda+2-b_{p+2} \\
2 n+\lambda+p+2, h+\lambda+1-a_{p}
\end{array} \mid 1\right), \\
& \rho_{n}:=\Gamma(2 n+\lambda+p+2) \sum_{m=p+2}^{\infty}(-1)^{m} \frac{m ! v_{m}(n)}{\Gamma(m+2 n+\lambda)} .
\end{aligned}
$$

Hence the result follows from (3.30).

E. The difference equation

$$
\sum_{m=0}^{p+1} G_{m}^{*}(n ; p+1) \Phi_{n-m}=0
$$


where the coefficients $G_{m}^{*}$ are defined by (3.6), is obviously equivalent to Eq. (3.20). Because of the symmetry property (3.7), Eq. (3.31) is invariant under the change of variable $n:=-n-\lambda$. Thus $R_{n}^{(p+1)}=$ const $\cdot R_{-n-\lambda}^{(p+2)}$ must also be a solution of (3.20).

F. As it has already been remarked, the functions (3.16)-(3.19) satisfy the difference equation

$$
\sum_{m=0}^{p+2} G_{m}(n ; p+2) \Phi_{n-m}=0
$$

of order $p+2$, and so any $p+3$ of them are linearly dependent. Thus the functions $S_{n}^{(k)}(k=1,2, \ldots, p+2)$ satisfy Eq. (3.20). This completes the proof of the theorem.

As a corollary to the above results, we give a difference equation satisfied by a family of functions, including $U_{n}$ as defined in (2.2).

Corollary. Let $n, q$ be integers $\geqslant 0$. Let $\beta, \lambda, c_{i}(i=1,2, \ldots, q+1), d_{j}(j=$ $1,2, \ldots, q)$ be complex constants such that none of the quantities $\beta+1, \lambda, c_{i}, d_{j}$ are negative integers or zero and that

$$
\operatorname{Re}\left(\sum_{i=1}^{q+2} c_{i}-\sum_{j=1}^{q} d_{j}-\lambda\right)<1
$$

where $c_{i}=\beta+1$ for $i=q+2$. Then the function

$$
U_{n}:=\frac{\left(c_{q+1}\right)_{n}}{\left(d_{q}\right)_{n}(n+\lambda)_{n}{ }_{q+2} F_{q+1}}\left(\begin{array}{c}
n+c_{q+2} \\
n+d_{q}, 2 n+\lambda+1
\end{array} \mid 1\right)
$$

satisfies the difference equation

$$
\sum_{m=0}^{q+1}(-1)^{m} H_{m}(n ; q+1) \Psi_{n+m}=0,
$$

the coefficients $H_{m}$ being given by (3.2) or (3.4). Further, if no two of the parameters $n$, $-n-\lambda, d_{j}(j=1,2, \ldots, q)$ differ by an integer or zero, then the functions

$$
\begin{aligned}
V_{n}:= & \frac{\omega_{n} \Gamma\left(n+\lambda+1-d_{q}\right)}{\Gamma\left(n+\lambda+1-c_{q+2}\right) \Gamma(1-\lambda-2 n)} \\
W_{n}^{(j)}:= & \frac{\omega_{n}}{\Gamma\left(2-d_{j}-n\right) \Gamma\left(n+\lambda+2-d_{j}\right)} \\
& \times_{q+2} F_{q+1}\left(\begin{array}{c}
c_{q+2}-n-\lambda \\
d_{q}-n-\lambda, 1-\lambda-2 n
\end{array} \mid 1\right), \\
2-d_{j}-n, n+\lambda+2-d_{j}, 1-d_{j}+d_{q+2}^{*} & \quad(j=1,2, \ldots, q)
\end{aligned}
$$

are also solutions of (3.33). Here

$$
\omega_{n}:=(2 n+\lambda) \Gamma(n+\lambda) / \Gamma(n+\beta+1) .
$$


Finally, if no two of the parameters $c_{i}(i=1,2, \ldots, q+2)$ differ by an integer or zero, then the functions

$$
\begin{aligned}
Z_{n}^{(k)}:= & \frac{(-1)^{n} \omega_{n}\left(c_{k}\right)_{n}}{\Gamma\left(n+\lambda+1-c_{k}\right) \Gamma\left(1+c_{k}-c_{q+2}\right)} \\
& \times_{q+2} F_{q+1}\left(\begin{array}{cc}
n+c_{k},-n-\lambda+c_{k}, 1-d_{q}+c_{k} \\
1+c_{k}-c_{q+2}^{*}
\end{array}\right) \\
& (k=1,2, \ldots, q+2)
\end{aligned}
$$

also satisfy Eq. (3.33).

Remarks. $1^{\circ}$. By virtue of a theorem in [8], the functions (3.32), (3.34)-(3.36) are solutions of (2.4). $2^{\circ}$. Making the substitution

in (3.33) yields the equation

$$
\Psi_{n}^{*}:=(\lambda-\beta)_{n} \Psi_{n} /(\lambda)_{n}
$$

$$
\sum_{m=0}^{q+1}(-1)^{m} H_{m}^{*}(n ; q+1) \Psi_{n+m}^{*}=0
$$

$H_{m}^{*}$ being defined by (3.8).

Proof. In (3.16)-(3.20), let $p:=q, a_{i}:=\lambda+1-d_{i}, b_{j}:=\lambda+1-c_{j}$. Then we have

$$
\begin{array}{cc}
R_{n}^{(j)}=C^{(j)} \tau_{n} W_{n}^{(j)} & (j=1,2, \ldots, p), \\
R_{n}^{(p+1)}=C^{(p+1)} \tau_{n} V_{n}, & R_{n}^{(p+2)}=C^{(p+2)} \tau_{n} U_{n}, \\
S_{n}^{(k)}=D^{(k)} \tau_{n} Z_{n}^{(k)} & (k=1,2, \ldots, p+2)
\end{array}
$$

for some constants $C^{(j)}, D^{(k)}$, and

$$
\tau_{n}:=(-1)^{n} \Gamma(n+1) \Gamma(n+\beta+1) /(2 n+\lambda) \Gamma^{2}(n+\lambda) .
$$

Now, in (3.20) make the substitution $\Phi_{n}:=\tau_{n} \Psi_{n}$, turn the sum around and replace $n$ by $n+p+1$. The result is

$$
\sum_{m=0}^{q+1} \tau_{n+m} G_{q+1-m}(n+q+1 ; q+1) \Psi_{n+m}=0 .
$$

However, a simple calculation shows that the identity

$$
\tau_{n+m} G_{q+1-m}(n+q+1 ; q+1)=\rho_{n}(-1)^{m} H_{m}(n ; q+1)
$$

holds for some rational function $\rho_{n}$ (cf. (3.2), (3.3)). Thus $\Psi_{n}$ satisfies (3.33).

4. Examples. In this final section we illustrate the application of the theorem by taking some examples of problems in which functions of the type (2.1) or (2.2) occur. These problems were originally solved using different methods. We show that the solutions can be readily obtained by applying the results of Section 3. Also, there are new implications in some places, which seem to be interesting.

A. Beta integral of the Jacobi function. In [2], the integral

$$
m_{n}:=\int_{0}^{1} x^{a-1}(1-x)^{b-1} R_{n}^{(\alpha, \beta)}(x) d x, \quad \operatorname{Re}(a)>0, \operatorname{Re}(b)>0,
$$

is examined. Here

$$
R_{n}^{(\alpha, \beta)}(x):=\frac{(\alpha+1)_{n}}{\Gamma(n+1)}{ }_{2} F_{1}\left(\begin{array}{c}
-n, n+\lambda \\
\alpha+1
\end{array} \mid 1-x\right) \quad(\lambda=\alpha+\beta+1)
$$


is the Jacobi function. If $n$ is an integer $\geqslant 0$, then (4.2) is the $n$th shifted Jacobi polynomial. (4.1) is called a beta integral of the Jacobi function. It can be shown that

$$
m_{n}=\frac{(\alpha+1)_{n} B(a, b)}{\Gamma(n+1)}{ }_{3} F_{2}\left(\begin{array}{c}
-n, n+\lambda, b \\
\alpha+1, a+b
\end{array} \mid 1\right) .
$$

The theorem of Section 3 yields the second-order recurrence relation

$$
\begin{aligned}
& n(n+\lambda-1)(2 n+\lambda-3)(n+a+b-1) m_{n} \\
&+(2 n+\lambda-3)_{3}[u(n)-u(n-1)-n-\lambda+b+1] m_{n-1} \\
&-(n+\alpha-1)(n+\beta-1)(2 n+\lambda-1)(n+\lambda-a-b-1) m_{n-2}=0, \\
& u(n):=(n+\beta)(n+\lambda-1)(n+\lambda-a-b) /(2 n+\lambda-1) .
\end{aligned}
$$

In a special case, when $b=1$, this theorem gives for the integral

$$
m_{n}^{*}:=\int_{0}^{1} x^{a-1} R_{n}^{(\alpha, \beta)}(x) d x,
$$

the first-order recurrence relation

$$
\begin{aligned}
(n+\lambda-1)(n+a) m_{n}^{*}+(n+\beta)(n+\lambda-a-1) m_{n-1}^{*} \\
\quad=(2 n+\lambda-1)(\alpha)_{n} / \Gamma(n+1) .
\end{aligned}
$$

These recursions can be used to evaluate (4.1) or (4.3), respectively. Note that in [2] (see also [9]), another computational scheme, based on a second-order recurrence relation for a certain auxiliary sequence, was used.

B. The Wilson polynomials. The $n$th Wilson polynomial is defined by [6], [7]

$$
\begin{array}{r}
p_{n}(t):=(a+b)_{n 4} F_{3}\left(\begin{array}{c}
-n, n+\lambda, a-t^{1 / 2}, a+t^{1 / 2} \\
a+b_{1}, a+b_{2}, a+b_{3}
\end{array} \mid 1\right) \\
\lambda=a+b_{1}+b_{2}+b_{3}-1 .
\end{array}
$$

In the sequel we use the notation $f(b)$ for $f\left(b_{1}\right) f\left(b_{2}\right) f\left(b_{3}\right)$. Thus, $(a+b)_{n}$ is short for $\left(a+b_{1}\right)_{n}\left(a+b_{2}\right)_{n}\left(a+b_{3}\right)_{n}$. Notice that the series ${ }_{4} F_{3}$ in (4.4) is balanced. The Wilson polynomials form an orthogonal system with respect to a positive measure on the real line, provided $a, b_{1}, b_{2}, b_{3}$ have positive real parts [7]. Application of our theorem yields the three-term recurrence formula

$$
\begin{aligned}
(n+\lambda) p_{n+1}(t)-(2 n+\lambda)_{2}\left[t-(n+a)^{2}-v(n)+v(n+1)\right] p_{n}(t) & -(n+\lambda-a-b)(2 n+\lambda+1) v(n) p_{n-1}(t)=0, \\
& -(n+a) /(2 n+\lambda-1),
\end{aligned}
$$

which agrees with Wilson's result [6]. For $a+t^{1 / 2}=1$ or $a-t^{1 / 2}=1$ we have the nonhomogeneous two-term relation

$$
(n+\lambda-1) p_{n}(t)-n p_{n-1}(t)=(a+b-1)_{n}(2 n+\lambda-1) .
$$

C. The Hahn polynomials. For real $\alpha>-1, \beta>-1$ and for positive integral $N$, the Hahn polynomials $Q_{n}(x)=Q_{n}(x ; \alpha, \beta, N)$ are defined by

$$
Q_{n}(x):={ }_{3} F_{2}\left(\begin{array}{c}
-n, n+\lambda,-x \\
\alpha+1,-N
\end{array} \mid 1\right) \quad(n=0,1, \ldots, N),
$$


where $\lambda:=\alpha+\beta+1$. The polynomials $Q_{0}, Q_{1}, \ldots, Q_{N}$ constitute an orthogonal system on $x=0,1, \ldots, N$ with respect to the measure

$$
(\alpha+1)_{x}(\beta+1)_{N-x} / x !(N-x) ! .
$$

The three-term recurrence relation for (4.5), obtained earlier by Weber and Erdélyi (see [3]), follows readily by application of the theorem of Section 3.

It is interesting that if $x=-1$ or $x=N+1$, then the Hahn polynomials satisfy a first-order nonhomogeneous equation. Namely,

$$
\begin{aligned}
& (n+\lambda-1)(n+\alpha)(n-N-1) Q_{n}(-1)+n(n+\beta)(n+\lambda+N) Q_{n-1}(-1) \\
& \quad=-\alpha(N+1)(2 n+\lambda-1),
\end{aligned}
$$

and, in view of [3],

$$
Q_{n}(N+1)=(-1)^{n} \frac{(\beta+1)_{n}}{(\alpha+1)_{n}} Q_{n}(-1),
$$

a similar result for $Q_{n}(N+1)$ holds.

D. Representation for ${ }_{3} F_{2}(1)$. Recently, Wimp [11] has shown that

$$
{ }_{3} F_{2}\left(\begin{array}{c|c}
\alpha_{1}, \alpha_{2}, \alpha_{3} \\
\beta_{1}, \beta_{2}
\end{array}\right),
$$

the parameters $\alpha_{i}, \beta_{j}$ being not interrelated, cannot be represented in closed form. Actually, he showed more by proving that the classical Watson formula (see [1, p. 189]; or [4, vol. 1, p. 104]) cannot be generalized. The main tool used in the proof was the difference equation

$$
\begin{aligned}
& \frac{(n+\lambda-1)(n+a)}{(2 n+\lambda-2)_{2}} \Phi_{n-1} \\
& \quad-\left[\frac{(n+\beta)(n+a-1)}{2 n+\lambda-1}-\frac{(n+\beta+1)(n+a)}{2 n+\lambda+1}+n+b-1\right] \Phi_{n} \\
& \quad-\frac{(n+\alpha+1)(n+\beta+1)(n+\lambda-a+1)}{(n+\lambda)(2 n+\lambda+1)_{2}} \Phi_{n+1}=0
\end{aligned}
$$

$\left(n+a\right.$ is short for $\left(n+a_{1}\right)\left(n+a_{2}\right)$, etc. $)$, satisfied by

$$
\begin{aligned}
C_{n}:=\frac{(a)_{n}}{(b)_{n}(n+\lambda)_{n}{ }_{3}} F_{2}\left(\begin{array}{c}
n+a_{1}, n+a_{2}, n+\beta+1 \\
n+b, 2 n+\lambda+1
\end{array} \mid 1\right), \\
\lambda=\alpha+\beta, \alpha>-1, \beta>-1,
\end{aligned}
$$

which follows by application of the corollary of Section 3. Wimp constructed Eq. (4.6) by using another technique. He also gave other solutions of this equation, corresponding to (3.34)-(3.36).

Acknowledgment. The author wishes to express his gratitude to Professor Jet Wimp for suggesting this investigation as well as for many helpful comments and critical remarks made during preparation of the paper. He also would like to thank the referee for his helpful suggestions. 
1. A. ERdEly et al., Higher Transcendental Functions, Vol. 1, McGraw-Hill, New York, 1953.

2. S. L. Kalla, S. Conde \& Y. L. LuKe, "Integrals of Jacobi functions," Math. Comp., v. 38, 1982, pp. 207-214.

3. S. Karlin \& J. L. MCGregor, "The Hahn polynomials, formulas and an application," Scripta Math., v. 26, 1961, pp. 33-46.

4. Y. L. LUKe, The Special Functions and Their Approximations, 2 vols., Academic Press, New York, 1969.

5. L. J. Slater, Generalized Hypergeometric Functions, Cambridge Univ. Press, Cambridge, 1966.

6. J. A. Wilson, "Three-term contiguous relations and some new orthogonal polynomials," in Padé and Rational Approximation (E. B. Saff and R. S. Varga, eds.), Academic Press, New York, 1977, pp. 227-232.

7. J. A. Wilson, "Some hypergeometric orthogonal polynomials," SIAM J. Math. Anal., v. 11, 1980, pp. $691-701$.

8. J. WIMP, "Recursion formulae for hypergeometric functions," Math. Comp., v. 22, 1968, pp. $363-373$

9. J. Wimp, “The computation of ${ }_{3} F_{2}(1)$," Internat. J. Comput. Math., v. 10, 1981, pp. 55-62.

10. J. WimP, “Differential-difference properties of hypergeometric polynomials," Math. Comp., v. 29 , 1975, pp. 577-581.

11. J. Wimp, “Irreducible recurrence relations and representation theorems for ${ }_{3} F_{2}(1)$," Comput. Math. Appl., v. 9, 1983, pp. 669-678.

12. S. Lewanowicz, "On the differential-difference properties of the extended Jacobi polynomials," Math. Comp., v. 44, 1985, pp. 435-441. 